\title{
New Species of Nyctibatrachus (Anura: Ranidae) from Castle Rock, Karnataka State, Southwest India
}

\author{
INDRANEIL DAS ${ }^{1,2}$ AND KRUSHNAMEGH KUNTE ${ }^{3}$ \\ ${ }^{1}$ Institute of Biodiversity and Environmental Conservation, Universiti Malaysia Sarawak, 94300, \\ Kota Samarahan, Sarawak, Malaysia; E-mail: idas@ibec.unimas.my \\ ${ }^{3}$ Section of Integrative Biology, University of Texas at Austin, 1 University Station C 0930 \\ Austin, Texas 78712,USA; E-mail: krushnamegh@mail.utexas.edu
}

\begin{abstract}
A new species of ranid frog of the genus Nyctibatrachus is described from Castle Rock, Karnataka State, Southwest India. The new species is compared with all known congeners and is diagnosed by the following combination of characters: head wider than long; snout projecting beyond mouth; supratympanic fold poorly defined; webbing on toes reaching base of disks except on Toe IV, where it reaches distal subarticular tubercle; tips of digits on fingers and toes flattened to form disks with distinct grooves separating dorsum of disks from venter; and femoral gland present. The largest specimen in the type series has a snout-vent length of $43.2 \mathrm{~mm}$. Nyctibatrachus hussaini Krishnamurthy, Reddy and Gururaja, 2001, is shown to be an invalid nomen, for lack of typification.
\end{abstract}

The genus Nyctibatrachus Boulenger, 1882 (type species: Nyctibatrachus major Boulenger, 1882, by subsequent designation of Myers, 1942) contains 11 nominal species currently considered valid (Duellman, 1993; Frost, 1985; Dutta, 1997; Glaw et al., 2000). Dubois (1987 "1986") allocated the genus to the subfamily Raninae, tribe Ranixalini, and subsequently (Dubois, 1992), to the subfamily Ranixalinae. Members of the genus are restricted to the Western Ghats, the interrupted mountain ridge that runs along the west coast of peninsular India, a region known for its distinctive amphibian fauna (see Inger and Dutta, 1986; Daniels, 1992, Biju, 2001; Biju and Bossuyt, 2003).

Species of Nyctibatrachus are recognizable by the following suite of characters: contracted pupil rhomboidal; habitus short, squat; omosternum and sternum with a bony style; tongue free, notched posteriorly; tympanum concealed; dorsum with longitudinal (transversely widened) skin folds; and tips of fingers and toes widened. In this paper, we describe a new species of Nyctibatrachus from Castle Rock, Karnataka State, Southwest India.

\section{Materials AND Methods}

The type series was fixed in formalin, and subsequently washed in water and transferred to $70 \%$ ethanol. The following measurements were taken with Mitutoyo ${ }^{\mathrm{TM}}$ dial vernier calipers (to the nearest $0.1 \mathrm{~mm}$ ), approximately 4.5 years after collection: snout-vent length (SVL, from tip of snout to vent); tibia length (TBL, distance between surface of knee to surface of heel, with both tibia

\footnotetext{
${ }^{2}$ Corresponding Author.
}

and tarsus flexed); head length (HL, distance between angle of jaws and snout-tip); head width (HW, measured at angle of jaws); head depth (HD, greatest transverse depth of head, taken posterior of the orbital region); eye diameter (ED, horizontal diameter of the eyes); interorbital distance $(\mathrm{IO}$, least distance between upper eyelids; internarial distance (IN, distance between nostrils); eye to snout distance (E-S, distance between anteriormost point of eyes and tip of snout); eye to nostril distance (E-N, distance between anteriormost point of eyes and nostrils); axilla to groin distance (A-G, distance between posterior edge of forelimb at its insertion to body to anterior edge of hind limb at its insertion to body); and body width (BW, greatest width of body). Color notes on live animals were taken from scanned images generated from slide transparencies.

Comparative materials examined are listed in Appendix 1. Sources of additional data on character states and distribution of congeneric species include the following works: Bhaduri and Kripalani (1954); Boulenger (1882); Chanda (2002); Chanda and Das (1997); Daniels (1997); Inger et al. (1984); Krishnamurthy et al. (1992, 2001); Pillai (1978); Rao (1920, 1922); Ravichandran (1997); and Shaffer (1988). Museum abbreviations, where available, follow Leviton et al. (1985).

\section{SYSTEMATICS}

Nyctibatrachus petraeus sp. nov. Figures 1-3

Type Series.-ZSI A.10070 (holotype) from Castle Rock, $15^{\circ} 25^{\prime} \mathrm{N}$; $74^{\circ} 19^{\prime} \mathrm{E}$, approximately 